Corresponding Author: Eleni

Andreou; email:

eandreou@eleniandreou.diet

Received 14 June 2021

Accepted 1 August 2021

Published 14 January 2022

Production and Hosting by Knowledge E

(c) Christiana Philippou, Eleni Andreou. This article is

distributed under the terms of

the Creative Commons

Attribution License, which

permits unrestricted use and redistribution provided that the original author and source are credited.

Editor-in-Chief:

Dr. Dimitrios Papandreou

Official Publication of Zayed University, UAE

\section{Integration of Healthy Eating Habits and Physical Activity through Nutrition Care Process to Tackle the Obesity Epidemic: A Narrative Review of the Evidence}

\author{
Christiana Philippou¹, Eleni Andreou²* \\ ${ }^{1}$ Department of Dietetics/Nutrition, University of Nicosia, Cyprus \\ ${ }^{2}$ European University of Cyprus, Cyprus \\ ORCID \\ Eleni Andreou: https://orcid.org/0000-0002-0449-6782
}

\section{Abstract}

Background: Obesity is a rising global health problem which is already at epidemic proportions Effective methods of treatment are required and should be imparted by efficient means to dietitians and other health professionals dealing with weight management. Research shows that behavioral modification techniques are the most effective way to achieve and maintain a healthy weight compared to diet and physical activity alone.

Aim: This narrative review focusses on diet and physical activity behavioral modification techniques to promote effective weight management for sedentary and active adults using the Nutrition Care Process (NCP).

Methods: PubMed, Scopus, Embase, Science Direct, Web of Science and Pro-Quest databases were searched for relevant articles.

Results: A healthy eating habit is one of the contributing factors to improved health. Physical activities also help improve and maintain one's health. This article discusses the importance of eating habits and physical activities among school students. In addition, health issues related to eating habits and the practice of physical activities are also highlighted. Overall, the results revealed that healthy eating habits and regular physical activities help in maintaining good health.

Conclusion: NCP is a systematic approach to provide high-quality nutrition care. Using the NCP does not mean that all clients get the same care. Use of a care process provides a framework for the dietitian to individualize care, taking into account clients' needs and values, and using the best evidence available to make decisions.

Keywords: obesity, weight control, physical activity, nutrition knowledge, eating habits, nutrition care process

\section{Introduction}

Obesity is a significant public health problem worldwide. According to the World Health Organization (WHO) statistics, in 2020, the global prevalence of overweight adults aged 18 years or older was $39 \%$. The number of obese and overweight adults is expected to 
reach 2.7 billion in 2025 [1]. Obesity is a complex, multifactorial condition that develops from social, behavioral, metabolic and physiological factors. Therefore, changing the environment can be associated with healthier eating behaviors, and interventions based on theories such as self-regulation have shown potential effectiveness [2].

Implementation of interventions is effective in improving the regular physical activity of adults and ensuring their health, as it can pave the way for the prevention of many diseases. The main focus of these interventions is weight management in adolescents by increasing regular physical activity. There is a need for a narrative review for the integration of eating habits and physical activity through the nutrition care process (NCP) in order to prevent and treat obesity. Studies have shown that interventions based on behavior change models and theory are more efficient to create a behavior change. Thus, the main objective of this narrative review was to assess the effect of educational interventions on promoting regular physical activity in adult weight management programs with the use of $\mathrm{NCP}[3,4]$.

\section{Methods}

\subsection{Search strategy}

PubMed, Scopus, Embase, Science Direct, Web of Science and Pro-Quest databases were searched for relevant articles. Using the population model, the intervention, comparison, results and design of the PICOS study were examined in papers published before the end of March 2020. The search strategy focused on three themes: participants (adults), outcomes (physical activity and weight management) and study type (all study design). The articles' references were searched for reassurance. The keywords derived from MeSH were "Obesity," "Weight Control," "Physical Activity," "Nutrition Knowledge," "Eating Habits" and "Nutrition Care Process."

\subsection{Article selection criteria}

To study the inclusion and exclusion criteria of the study, the PICOS index (population under study, type of studies, types of interventions and type of outcome) was used. The population under study was the adult age group. The types of interventions included interventions conducted with the use of NCP. The study design of all included studies were weight management interventions and increase of physical activity in different places. No publication date limitation was set and only studies published in English were included. At first, all studies were reviewed based on the proposed search strategy, 
and all obtained articles were entered into the EndNote software. Duplicate items were then removed in the EndNote. The title and summary of the relevant studies were independently examined in terms of relevance to the research question.

\subsection{Obesity}

The worldwide incidence of obesity is increasing. In fact, a new word - "globesity" has now emerged to reflect the increase of global obesity and overweight. In 1998, the WHO published a report entitled, "Obesity: Preventing and Managing the Global Epidemic," which classified obesity as a rising epidemic. According to the WHO, if no prompt action is taken, millions will suffer from serious weight-related illnesses [5]. Obesity is universal in the contemporary society that health professionals report this issue on a regular basis [6-8] . Obesity can be defined as an abnormal or excessive fat accumulation which presents a risk to health. It can also be defined using a person's body mass index (BMI) - the person's weight $(\mathrm{kg})$ divided by the square of their height $(\mathrm{m})$ - where a BMI of 30 is considered obese and 25 overweight. Moreover, obesity can be classified into three classes based on its severity: Class I (BMI of $30.0-34.9 \mathrm{~kg} / \mathrm{m}^{2}$ ), Class II (BMI of $35.0-39.9 \mathrm{~kg} / \mathrm{m}^{2}$ ) and Class III (BMI of $\left.\geq 40.0 \mathrm{~kg} / \mathrm{m}^{2}\right)[5,8,9]$.

Overweight and obesity are major risk issues for many chronic diseases, including diabetes, CVD and cancer. Once considered a problem only in high-income countries, overweight and obesity are now dramatically on the rise in low- and middle-income countries, particularly in urban settings [11, 12].

Currently, the number of overweight people around the world rivals the number of underweight people. Some developing nations have also been troubled with obesity. A 1999 United Nations (UN) survey found obesity increasing in all developing regions, even in countries affected by hunger. In China, the number of overweight people rose from $<10 \%$ to $15 \%$ in only three years. In Brazil and Colombia, about $40 \%$ of the population is overweight, similar to several European countries. Also, sub-Saharan Africa, where most of the world's hungry live, is perceiving an increase in obesity, particularly among urban women. In all regions, obesity seems to heighten with a rise in income. In the United States, obesity is the most common chronic disease, affecting more than one in every four American and its frequency has been progressively increasing for the past 20 years. In Europe, Australia/New Zealand, the Middle East and the remaining parts of the Americas, the occurrence of obesity seems to be increasing to 10-20\%. The prevalence of obesity is however relatively low in China, Japan and several countries in Africa. Table 1 shows the prevalence of obesity worldwide. These increasing figures alarm dietitians and health professionals for the need of effective methods of 
TABLE 1: Percentage of obese adults in Europe, Australia and USA

\begin{tabular}{l|c|c|}
\hline Country & $\begin{array}{c}\text { Percentage of obese } \\
\text { men }\end{array}$ & $\begin{array}{c}\text { Percentage of obese } \\
\text { women }\end{array}$ \\
\hline Finland (2005) & 14.4 & 19.3 \\
\hline Russia & 10.8 & 27.9 \\
\hline England & 17 & 20 \\
\hline Germany & 17.2 & 19.3 \\
\hline Czech & 16.3 & 20.2 \\
\hline Scotland & 15.9 & 17.3 \\
\hline Belgium & 12.1 & 18.4 \\
\hline Spain & 11.5 & 15.2 \\
\hline Sweden & 10 & 11.9 \\
\hline France & 9.6 & 10.5 \\
\hline Denmark & 10 & 9 \\
\hline Holland & 8.4 & 8.3 \\
\hline Italy & 6.5 & 6.3 \\
\hline Cyprus (1999-2000) & 26.6 & 23.7 \\
\hline Cyprus (2009) & 28.8 & 26.9 \\
\hline Greece (2003) & 26 & 18.2 \\
\hline Australia & 18 & 18 \\
\hline USA & 31.1 & 33.2 \\
\hline Source: [12, 13] & & \\
\hline & & \\
\hline & & \\
\hline & &
\end{tabular}

treatment to deal with weight management. In fact, diet and physical activity behavioral modification techniques promote effective weight management for sedentary and active adults [12].

\subsection{Theory of behavioral modification}

Behavior modification indicates the method used to decrease or increase a specific target behavior or reaction. While parents use this to communicate to their children what is right and what is wrong, counsellors use it to encourage healthy behaviors in their patients [14].

Diet and exercise alone, or diet and exercise or diets with appetite-suppressants generally result in insignificant weight loss with fast weight regain [15]. The results are better when a behavior modification component is introduced with any of these weight loss strategies. The term "behavior modification" might be a paired term, since successful weight-loss behavior modification aims at reducing calorie intake, increasing physical activity and expanding the nutrition knowledge and food choices for an indefinite period. Although some people can achieve this alone, most cannot. Compliance 
with behavior and lifestyle modifications to lose weight and maintain weight loss can be extremely difficult because of genetic background, environmental pressures and ingrained life-long behaviors. Martin's research indicated that women more than men were prone to fail in their weight management (failure rate: $44 \%$ female, $29 \%$ male) and there was a high incidence of eating disorders (binge eating and/or extreme weight control) [14].

Every study discussing "predictors" of successful weight loss shows that exercising for at least $30 \mathrm{~min} /$ day at least 4 days/wk is vital. Engaging in regular physical activity is an even stronger predictor of successful long-term weight maintenance and has the additional advantage of improving cardiovascular fitness [16]. The health benefits of eating habits and regular physical activity are well-known. Regardless of the recommendation to be used, the health benefits of regular physical activity are well-established [17].

The preferred therapy for overweight and obese patients is a mix of diet, exercise and behavior modification [18]. The first guidelines for obesity were initiated by the Federal Government in USA to address overweight and obesity conditions that affect an approximately 97 million Americans and are the second leading cause of death, which can be prevented, in the United States. These evidence-based guidelines, developed by the National Heart, Lung, and Blood Institute in cooperation with the National Institute of Diabetes and Digestive and Kidney Diseases, present an approach for the valuation of overweight and obesity and create values of safe and effective weight loss.

\subsection{Nutrition Care Process (NCP)}

Giving excellent nutrition care implies making the best choice at the ideal time, in the correct way, for the right individual and accomplishing the most ideal outcomes. Studies show that when a standardized process is executed, not so much variety but rather more consistency, the results are better. A standardized NCP effectively helps dietitians as the sole nutrition provider of sustenance care when it is reliably utilized as a methodical technique to think and settle on choices to give protected and compelling nutrition care. The NCP is presented in Figure 1 [19] while Figure 2 presents the Critical Path for the Nutritional Management of Obesity.

(i) Nutrition Assessment and Reassessment: The RDN accumulates and records proof, for example, food- or nutrition-related history; biochemical information, clinical tests and strategies; anthropometric measurements, nutrition findings and patient's history. 


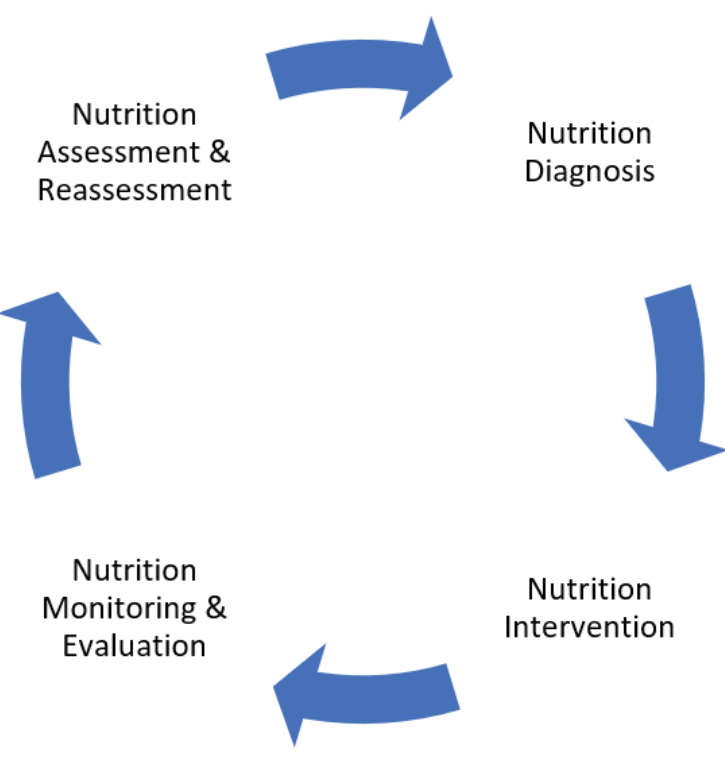

Figure 1: The Nutrition Care Process.

(ii) Nutrition Diagnosis: Data gathered during the nutrition assessment directs the RDN in determination of the proper nutrition diagnosis(es) (i.e., naming explicit issues) terms.

(iii) Nutrition Intervention: The RDN at that point chooses the nutrition intervention(s) that will be coordinated to the underlying etiology and additionally pointed toward mitigating the signs and symptoms of each diagnosis.

(iv) Nutrition Monitoring/Evaluation: The last step of the process is monitoring and evaluating, which the RDN uses to decide whether the patient has accomplished, or is gaining ground toward, the planned goals.

NCP provides dietitians with the revised "road map" to go along the best path for highquality patient/client/group-centered nutrition care. The critical path for the nutritional management of obesity is presented in Figure 2.

In a recent research, scientists have shown that higher inspiration could lead social change for obese children and teenagers. They assessed the impact of the NCP on the consumption of calories and weight status in moderate-to-serious obese kids and young people in Korea. One hundred and four participants (mean age: 10.95 years, BMI $\geq 97^{\text {th }}$ percentile) took an interest in the research. Participants were divided into two groups: a usual care group (UG) and a nutrition group (NG). All participants went through nutrition education six times. The NG got singular access and nonstop observing and defining objectives concerning health issues. Utilization of unhealthy, low-supplement (HCLN) food was altogether reduced $(P<0.05)$ while the Diet Quality Index-International (DQII) score concerning sodium additionally increased $(P<0.001)$. The absolute self-viability 


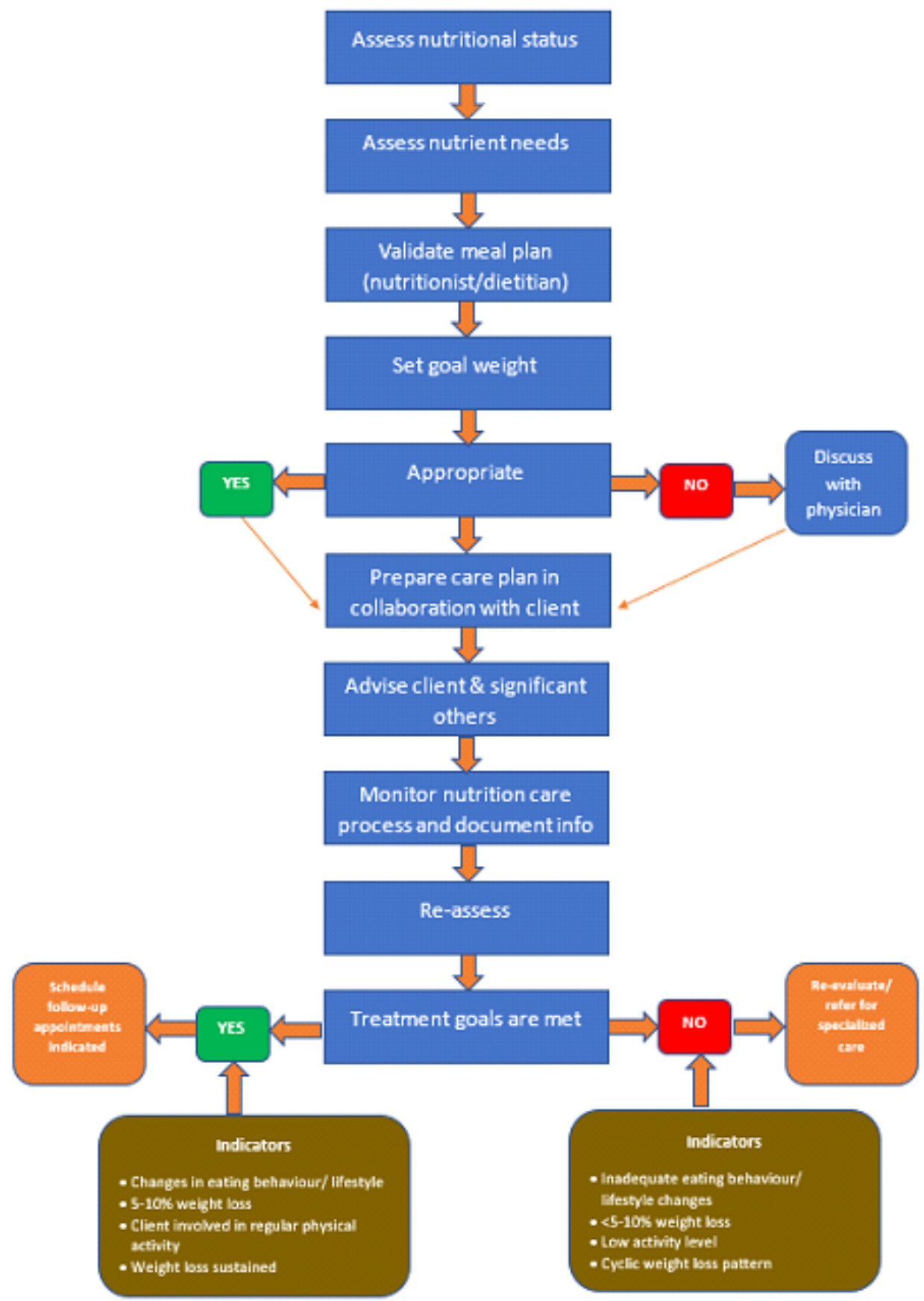

Figure 2: Critical path for the nutritional management of obesity (NCP).

score was increased from 9.15 to $10.14(P<0.01)$. Following 24 weeks, the BMI-z-score decreased from 2.27 to 2.19 in the NG $(P<0.05)$; however, no group differences was found. BMI-z-score was adversely connected with self-viability $(\square=-0.03, P<0.019$ ). The results indicate that the NCP-based intervention may help tackle dietary issues by improving self-adequacy and lower BMI-z-score in seriously obese youngsters [20].

In another study, researchers showed how phone and computer applications (apps) could enhance the efficiency of the NCP. The aim of this study was to provide dietitians 
with practical direction on including smartphone apps in the NCP to enhance patient education and counselling. The current evidence-based mobile health (mHealth) apps examine where and how apps could be applied by dietitians through all the four steps of the NCP. The results show the necessity for an immediate change in the patient's dietary records into nutrient substances, where nutrition assessment can be restructured using nutrition applications, permitting extra time for dietitians to offer education and nutrition consultation. Dietitians could recommend applications to educate patients on the nutrition skills for behavioral change. For efficient patient-dietitian communication, the actual overview and assessment of patient improvement could be achieved using applications. A pragmatic outline characterized as "Mobile Nutrition Care Process Grid" guided the dietitians on the usage of the applications. The effectiveness of the nutrition care and consultation provided by dietitians could be reinforced with the usage of good-quality applications [21].

\subsection{Behavioral modification approaches for weight management}

Behavioral approaches to obesity are based on two assumptions: first, eating and exercise behaviors are related to body weight, and second, behaviors can be modified by changing both the antecedents, or cues in the environment, that come before the behavior and lead to its occurrence, and the consequences, or reinforcements, that come after the behavior and increase its frequency [22]. Based on these premises, there are three main components to a behavioral approach (physiological, cultural and past or immediate environment) designed to assess the behaviors and change the antecedents and consequences controlling the behaviors. In order to determine the behaviors that need to be changed and assess progress in making these changes, it is necessary to find a way to monitor behavior. In weight loss programs, eating and exercise behaviors are usually self-monitored. Patients are asked to write down all food items consumed by them and all leisure physical activities that were accomplished. Although these reports may underestimate the intake or overestimate the activity [23], they can be used by the patients and therapist to identify specific problem areas (e.g., is the participant consuming large portion sizes, selecting high fat choices etc.?) and to estimate progress. According to Lichtman et al., some obese people continually fail to lose weight even though they state that they restrict their energy intake to $<1200$ $\mathrm{kcal} / \mathrm{day}$ [23]. They studied two reasons for this obvious battle to diet: (a) low total energy expenditure and (b) under reporting/estimating of caloric intake. Group 1 included nine women and one man with a record of diet resistance in whom they assessed the total energy spending and actual energy intake for 14 days by indirect calorimetry 
and analysis of body composition. Group 2 (control group) consisted of 67 women and 13 men with no history of diet resistance. The results of this study showed that the total energy expenditure and resting metabolic rate in the participants with diet resistance were within $5 \%$ of the expected values for body composition, and there was no significant difference between the two groups in the thermic effects of food and exercise. On the other hand, the subjects in group 1 understated their real food intake by an average $( \pm S D)$ of $47 \pm 16 \%$ and overstated their physical activity by $51 \pm 75 \%$. Although the participants in the first group had no clear psychopathologic attributes, they identified a genetic cause for their obesity, increased use of thyroid medication, and reported their eating behavior as relatively usual (all $P<0.05$ as compared with group 2). The study showed that the failure of some obese subjects to lose weight while eating a low-calorie diet was due to an energy intake considerably greater than described and an overrating of physical activity.

Behavioral approaches assume that the environment is an important determinant of behavior. Especially, the physical environment, including the sight and smell of food, can activate moods of hunger and influence what type of food is selected. Other types of environmental signals can also be significant. Eating and exercise behaviors can be influenced by social (the behaviors or attitudes of others around the patient) and cognitive cues (thoughts and feelings about eating, exercise and body weight). Thus, behavioral approaches include techniques to change physical, social and cognitive cues. The third key component of a behavioral program is increasing reinforces for new, appropriate behaviors. Patients are taught to recognize small positive changes in their behavior and reward themselves verbally and with small tangible rewards for this progress. Therapist praise and social support from others in the treatment program are also used as reinforcement.

Behavioral approaches are used to help patients make long-term changes in their eating and exercise behaviors [24]. To achieve this, behavioral approaches underline observing dietary intake and physical activity and altering the signs and support in the environment. Better results have been achieved in behavioral programs that provide longer periods of treatment contact, more structured approaches to modifying dietary intake and higher goals for physical activity. The current practice guidelines for the management of overweight and obesity recommend a program of diet, exercise and behavior therapy for all persons with a BMI of a minimum of 30 (and those with BMI $\geq 25$ along with two weight-related comorbidities). This lifestyle modification-behavior therapy treatment gives a structure that facilitates meeting goals for energy intake and expenditure. Even if a standard behavior therapy reliably leads to a weight loss of roughly $10 \%$ of initial weight, these reductions are difficult to maintain. Fabricatore et al. 
[25] claim that a shift from behavior change to cognitive change will improve long-term outcomes of programs that emphasize lifestyle changes. This study depicts the standard behavioral treatment (SBT) of obesity and relates it with an alternative treatment model which is based on a cognitive conceptualization of weight control.

Self-monitoring is the core of all behavioral programs and consists of self-observation and self-recording of those observations. The situational factors, behaviors, thoughts and feelings that occur before, during and after attempts at prudent eating and exercise behaviors are recorded. Self-watching may avoid improper behavior because the patients know that their documented indiscretions will be examined by a counsellor [26]. Studies by Perri et al. [27] found that patients spontaneously reduced calorie intake when daily diet records were kept. Patients who monitored their calorie intake and expenditure lost more weight than those who did not use self-monitoring. Several studies have found good correlations between self-monitoring and weight loss $[27,28]$ and maintenance $[29,30]$ although self-monitoring and success could both be caused by a third motivational factor [26]

Stimulus control involves the modification of cues leading to inappropriate eating or inadequate exercise. Early theories suggested that obese persons were particularly sensitive to environmental cues and less sensitive than nonobese persons to internal, hunger cues [31]. Later research indicated that such sensitivity is not confined to obese persons and that not all obese persons are particularly sensitive [32]. Although few studies have compared behavioral treatments with and without stimulus control, the concept of controlling the environment is widely accepted as clinically effective. Control of food cues may help eating management because introduction to such cues has been shown to produce physiologic reactions such as insulin shifts, which may predispose one to overeat [33].

Contingency management was used to involve the application of rewards for appropriate behavioral patterns leading to weight loss or maintenance (e.g., buying a clothing accessory for achieving the weight goal). Contracts are used to formalize agreements. Contracts are short term and should focus on increasing healthful behaviors associated with weight loss rather than on weight loss itself. The success of contingency management frequently ends when the rewards end. Originally, changes in eating and exercise habits may be fundamentally aversive in such cases, false prizes for obedience are needed. Later, as the new eating and exercise behaviors become apparent as enjoyable and intrinsically rewarding, the contracts can be allowed to expire [34].

Modifications in behavior through change of the speed or intensity of target behaviors may be needed to improve outcome. After all, reductions in the rate of eating in response to behavior therapy have been positively correlated with weight loss in 
the short term [35]. Gradual modification of eating behaviors away from dieting and meal skipping toward more normal eating patterns (three meals a day) using a gradual substitution of lower-fat alternatives may be required to prevent emotions of deprivation that could cause lapses in eating control. Similarly, healthy physical activity habits will be established progressively to allow for cardiorespiratory adaptation and avoid the person's perception that physical activity is a punishment.

Cognitive-behavioral strategies will be used to help move thinking patterns away from self-rejection and toward self-acceptance. The focus should be on the ways in which thoughts, moods, diets and social pressure to be thin affect eating control. About $40 \%$ of all obese patients seeking treatment suffer from binge-eating disorder, characterized by frequent and uncontrollable episodes of binge eating [36]. Cognitive-behavioral treatment for binge eating has been shown to be effective [37] and may need to precede behavioral treatment of obesity. An approach using cognitive-behavior treatment to reduce restrictive dieting appears to have alleviated much of the psychological distress associated with obesity [26].

Recent study provides behavioral strategies for working with obese patients and families within a primary care context. Multicomponent programs include a combination of nutritional, physical activity, and cognitive behavioral approaches to target overweight/obesity. The focus is on behavioral approaches and practical applications, such as motivational interviewing techniques [38].

In Philippou's (2011) study, the main goal was to determine for the first time the effects of a weight-loss diet with or without exercise on body fat (BF) percentage and other anthropometrics in overweight and obese Cypriot adults [39]. The results showed no significant differences in both groups (diet/exercise [DE] and diet only [DO]) during the baseline period. Throughout the intervention period, weight, BMI and waist circumference (WC) decreased significantly in the DO group $(P<0.001)$. In addition, BF levels were found to be significantly reduced $(P<0.001)$ in the DE group compared with the DO group. In multiple regression analysis, BF levels were independently associated with weight (Beta: 0.569, 95\%Cl: 0.157-1.296, $P<0.001$ ), BMI (Beta: 0.295, 95\%Cl: 0.088-1.214, $P<0.01$ ) and WC (Beta: 0.206, 95\% Cl: 0.095-0.954, $P<0.001$ ) after adjustment of age and gender. Despite the reduced effects of diet alone on body weight, BMI and WC, combining a reduced diet with exercise also improves BF levels which may have important benefits in overcoming several diseases later in life. 


\subsection{Diet and eating habits for weight loss and maintenance}

Behavioral programs attempt to change energy balance by influencing both the calorie intake and expenditure. Most programs emphasize on decreasing overall calorie intake and restricting fats specifically. At the start of the program, participants are assigned a calorie goal designed to produce a weight loss of $1-2 \mathrm{lb} / \mathrm{wk}(0.5-1 \mathrm{~kg} / \mathrm{wk})$. This is done on individual basis by estimating their current calorie intake and then deducting 500-1000 $\mathrm{kcal} / \mathrm{day}$. In some studies, the current intake is estimated by multiplying the patients' weight in pounds (Ibs) by 12, and in others resting energy expenditure is estimated and an adjustment made for the patient's activity level. Alternatively, in many programs, participants are simply assigned a calorie goal depending on their initial body weight (e.g., patients weighting $<200 \mathrm{lb}[<91 \mathrm{~kg}]$ may be asked to eat $1000-1200 \mathrm{kcal} /$ day and those weighing $>200 \mathrm{lb}[>91 \mathrm{~kg}]$ may be asked to eat $1500 \mathrm{kcal} /$ day). Very low-calorie diets (VLCD; diets <800 kcal/day) were extremely popular in the 1980s [40].

The NTFPTO study aimed to provide an overview of the published scientific information on the safety and efficacy of VLCDs and to provide rational recommendations for their use [40]. VLCDs were usually provided in the context of comprehensive treatment programs, during which usual food intake is completely replaced by specific foods or liquid formulas containing $3350 \mathrm{~kJ} / \mathrm{d}(800 \mathrm{kcal} / \mathrm{d})$ or less. Weight loss on VLCDs averages $1.5-2.5 \mathrm{~kg} / \mathrm{wk}$; total loss after 12-16 weeks averages $20 \mathrm{~kg}$. These results are superior to standard low-calorie diets of $5020 \mathrm{~kJ} / \mathrm{d}(1200 \mathrm{kcal} / \mathrm{d})$, which lead to weight losses of $0.4-0.5 \mathrm{~kg} / \mathrm{wk}$ and an average total loss of only $6-8 \mathrm{~kg}$. There is little evidence that intakes of $<3350 \mathrm{~kJ} / \mathrm{d}(800 \mathrm{kcal} / \mathrm{d})$ result in better weight losses than those of $3350 \mathrm{~kJ}$. Consumption of at least $1 \mathrm{~g} / \mathrm{kg}$ (IBW) per day of protein of high biological value seems to be crucial to help maintain lean body mass. Serious complications of modern VLCDs are unusual, cholelithiasis being most common. Lastly, VLCDs are usually safe when used under proper medical supervision in moderately and severely obese patients $(\mathrm{BMI}>30)$ and are typically effective in promoting significant short-term weight loss, with improvement in obesity-related conditions. Long-term maintenance of weight lost with VLCDs is not very satisfactory and is no better than with other forms of obesity treatment. Combination of behavioral therapy and physical activity in VLCD treatment programs appears to improve maintenance. These diets are usually consumed as liquid formula or lean meat, fish and fowl. Patients have lost an average of $9 \mathrm{~kg}$ in 12 weeks on these treatments. However, after stopping the diet, regain was common. Therefore, several studies were designed to examine the combination of VLCDs and behavior modification, reasoning that VLCD might increase initial weight loss and behavioral approaches might improve maintenance of weight loss [41]. The combination of behavior 
modification plus VLCD was found to be more effective than VLCD alone. However, behavior modification plus VLCD was not more effective than behavior modification with a low-calorie diet (1200-1500 kcal/day). Combining behavior modification with a VLCD increased initial weight loss, but despite the behavioral training, these patients regained great amounts of weight over the year of follow-up. Given these results, along with concern regarding health consequences of rapid weight loss, the expense of using VLCDs and the evidence that weight losses are comparable on liquid diets of 400 , 600 and $800 \mathrm{kcal} / \mathrm{day}$, most weight loss programs now use higher calorie levels (>800 $\mathrm{kcal} / \mathrm{day})$. Many behavioral programs encourage patients not only to reduce their overall calories but also to lower their fat intake to $20-30 \%$ of their calories to improve weight loss and lipid responses. The combination of restricting dietary fat and calories has been shown to be more effective than fat restriction alone [42] or calorie restriction alone [43].

Schlundt examined a low fat with a free access to carbohydrate diet for weight reduction [42]. Fifty-two females and eight males (60 total) were randomized to low fat with free feeding to carbohydrate (low-fat) or low fat with caloric control (low-calorie) behavior modification treatments. Subjects in both groups stated an average of over five exercise sessions per week during treatment. The low-calorie group lost significantly more weight (males $11.8 \mathrm{~kg}$, SD 6.4; females $8.2 \mathrm{~kg}$, SD 4.2) than the low-fat group (males $8.0 \mathrm{~kg}$, SD 1.3; females $3.9 \mathrm{~kg}$, SD 3.7). Both groups lost analogous amounts of lean body mass. There was considerably greater loss of BF in the low-calorie group. Fat intake was decreased from 90 to $30 \mathrm{gr}$ per day. Subjects in both groups decreased their total energy intake with the low-calorie group consuming fewer calories per day than the low-fat group. Both groups showed important and comparable progresses in eating habits derived from nutrient analysis of eating diaries. Nevertheless, eating socially and emotionally continued to cause obedience problems during treatment for both groups. On the other hand, Hays et al. [44] in another study showed that a highcarbohydrate diet (18\% fat, 19\% protein, 63\% carbohydrates and $26 \mathrm{gr}$ of fiber per 1000 kcal) consumed at free access, with no effort at calorie control or alteration in energy consumption caused weight loss and BF reduction in older men and women.

Another study investigated the effects of a behavioral weight loss program emphasizing calorie restriction against calorie and fat restriction in obese individuals with type-2 diabetes or a family history of diabetes [43]. The aim of this randomized study was to compare the effects of a behavioral intervention focusing on calorie restriction alone or calorie and fat restriction on weight loss and deviations in lipids and glycemic control in individuals with non-insulin-dependent diabetes mellitus (NIDDM) or a family history of diabetes. The study involved 44 obese women with NIDDM and 46 obese women with 
a family record of NIDDM who were at random given put to calorie restriction (CAL) or calorie plus fat restriction (CAL + FAT). All subjects participated in a 16-week behavioral weight loss program, with training in diet, exercise and behavior modification. Subjects appointed to the CAL group were given a 1000-1500 kcal/day target and were asked to self-monitor the calories eaten. Subjects assigned to the CAL + FAT group had the same calorie goal but were also given a fat goal (grams of fat/day) to produce a diet with $<20 \%$ of energy from fat; this group checked both calories and fat. For the NIDDM subjects, weight loss in the CAL + FAT group was significantly higher than in the CAL group ( 7.7 vs $4.6 \mathrm{~kg}$ ); the former group also showed better results in maintaining their weight loss at the one-year follow-up (5.2 vs $1.0 \mathrm{~kg}$ ). Substantial reductions in glucose, low-density lipoprotein (LDL) cholesterol and total cholesterol were noted after 16 weeks of treatment including in type-2 diabetes participants; these alterations were analogous in CAL and CAL + FAT groups. At the on-year follow-up, all factors had returned to baseline. Minor changes in weight loss or physiological changes were seen between CAL and CAL + FAT conditions in subjects with a family history of diabetes. These results proposed that using the mixture of calorie and fat restriction may encourage weight loss in obese NIDDM patients. There were no other prolonged advantages of this treatment.

Moreover, reducing fat intake and decreasing consumption of specific high-fat food have been shown to be related to maintenance of weight loss $[45,46]$.

Holden et al. [45] studied the long-term follow-up of patients attending a combination of a very-low calorie diet and behavior therapy weight loss program. They studied the consequences of therapy with VLCD linked with behavior modification on weight loss and long-term maintenance of weight loss in 118 of 199 patients who completed eight weeks of VLCD. Harris et al. [46] examined the dietary and physical activity correlates of long-term weight loss. They examined alterations in BMI, physical activity, macronutrient intake and the frequency of consumption of foods among 82 men and 75 women participating in a behavioral weight loss program over a period of 18 months. Outcomes of repetitive procedures analyses of covariance revealed that BMI shift was inversely linked to change in physical activity and change in frequency of vegetable consumption. BMl change was positively related to the alteration in calorie intake from fat and modification in frequency of consumption of beef, hot dogs and sweets. Change in calories coming from fat predicted changes in BMl better than change in total calories. In addition, change in the frequency of consumption of specific foods accounted for a larger percentage of the variance in BMI change than did the change in macronutrients $(10.4 \%$ vs $5.2 \%$ ). There were no differences between predictors of weight loss as opposed to weight maintenance. For simplicity, participants are given 
a fat goal in grams of fat/day (e.g., participants on a $1200 \mathrm{kcal}$ diet are instructed to consume $27-40$ gr of fat for a diet of $20-30 \%$ fat).

Wing and Hill [47] described successful long-term weight loss and maintenance as the intentional loss of at least $10 \%$ of initial body weight and maintaining it for at least one year. Based on this definition, $>20 \%$ of overweight/obese persons were successful on their weight loss process. In the National Weight Control Registry, successful longterm weight loss maintainers (average weight loss of $30 \mathrm{~kg}$ for an average of 5.5 years) shared common behavioral strategies, as well as consuming a low-fat diet, regular selfexamination of body weight and food intake, and high levels of repeated physical activity. Once these successful maintainers maintained their weight loss for two to three years, the chances of longer-term achievement greatly increased. These individuals stated that they continued to eat a low-calorie (1380 kcal/day) and low-fat ( $24 \%$ of calories from fat) diet. Almost $80 \%$ of the participants confirmed eating breakfast every day during the week and only $4 \%$ reported never eating breakfast. Despite the recent popularity of diets recommending low carbohydrate intake, $<1 \%$ of registry participants reported consuming $<24 \%$ of their diet as carbohydrate

( $<90 \mathrm{gr}$ of carbohydrate on a $1500 \mathrm{kcal}$ regimen).

To identify the behavior-change strategies that were most evidently related toweight loss, 106 patients with type-2 (non-insulin-dependent) diabetescompleted the Eating Behavior Inventory (EBI) before and afterparticipating in a behavioral weight-loss program and at one-year follow-up [48].The EBI is a standardized questionnaire that assesses behavioral strategiestypically taught in a behavioral weight-loss program. Pretreatment scoreson the EBI were not related to weight-loss outcome, however, changes on the EBlin the direction of more frequent use of appropriate strategies wererelated to weight loss at both posttreatment and one-year follow-up. Specificstrategies related to weight loss at both times were: consuming foods thathelp in losing weight, recording foods eaten, refusing food offeredby others and being able to stop eating when appropriate. Nevertheless, fewpatients maintained frequent use of these strategies at follow-up. It isconcluded that weight-loss programs should give emphasis on the plans moststrongly linked to weight loss and attempt to improve long-term use of thesemethods.

Patients in behavioral weight loss programs were stimulated to select foods that provided the highest nutritional benefit for the least calories. The goal was to decrease the overall fat intake. Plans are provided for improving quality of foods consumed at home and when eating out. For example, patients are taught to substitute lower-calorie items for higher-calorie alternatives, restrict the use of fat in cooking and flavoring of foods and modify favorite recipes for healthier eating. Furthermore, providing increased structure 
regarding diet was considered an issue for behavioral modification in eating habits and exercise. There have been several studies suggesting that providing structure to patients on what they should eat, and thereby simplifying choices, preparation time, etc., could be very helpful in promoting dietary adherence $[49,50]$. Jeffery et al. [51] presented ways to strengthen behavioral interventions for weight loss: a randomized trial of food provision and monetary incentives. Behavioral treatments for obesity pursue to alter eating and exercise behaviors by a change in their experiences and consequences. More direct modification of experiences and consequences by (a) the provision of food to patients and (b) the provision of financial rewards for weight loss was hypothesized to improve treatment outcomes. No treatment was assigned randomly to 202 men and women, SBT, SBT and food provision, SBT and incentives, or SBT and food provision and incentives. The major finding of this study was that food provision significantly enhanced weight loss. Weight losses with SBT averaged 7.7, 4.5 and $4.1 \mathrm{~kg}$ at 6, 12 and 18 months, respectively, compared with 10.1, 9.1,and $6.4 \mathrm{~kg}$, respectively, at the same intervals with the addition of food. Food provision also enhanced attendance, completion of food records, quality of diet and nutrition knowledge. The conclusion of this study was that the providing of food to weight-loss people was a promising practice that deserved additional survey. Weight-maintenance studies applying financial incentives are very rare, so that the long-term impacts on public health benefits cannot be concluded [34].

Meta冈analyses with prospective studies were identified from searches in PubMed and Psyc INFO from 2006 to 2016. The articles investigated overweight and obese adults undergoing weight loss without surgery or medication. The search resulted in 8222 articles of which 67 were selected. In total, 124 determinants were identified of which 5 were demographic, 59 behavioral, 51 psychological/cognitive and 9 social and physical environmental determinants. That results showed that demographic factors were not predictive of weight-loss maintenance. Behavioral and cognitive determinants that promote a reduction in energy intake, an increase in energy expenditure and monitoring of this balance are predictive determinants. This review identifies key determinants in weight loss maintenance [52].

\subsection{Physical activity, weight loss and maintenance}

Several randomized controlled trials have compared the effects on weight loss of diet only, exercise only and the combination of the two [18]. These studies indicated that exercise alone has very little impact on body weight, and that combining exercise, diet and behavioral modification program increases initial weight loss by approximately $2 \mathrm{~kg}$. These small impacts of exercise may have been due to the low frequency of 
exercise used in many of these trials and the short length of the studies. The utmost benefits of exercise are seen in the maintenance of weight loss. Of the six studies that have studied long-term weight losses in diet only against diet combined with exercise, all six discovered that the latter had better outcomes, although there was no statistically significant difference [49]. Correlational data are even stronger in suggesting the benefits of long-term physical activity for the maintenance of weight loss [53].

Wing et al. [47] and Tate et al. [54] studied how physical activity contributed to the treatment of the adulthood overweight and obesity. This study evaluated the impacts of a 16 week of energy constraint and vigorous exercise on body mass and body composition. Sixty sedentary men with a mean age of 42.4 (5.0) years, mean body mass (mean \pm SD) 96.3 (13.9) kg were randomly designated to either sustain their normal energy intake or limit energy consumption by $4186-6279 \mathrm{~kJ} /$ day. Each group was further randomized to a control low-moderate intensity exercise program or a vigorous exercise program for three half-an-hour sessions per week. Strong exercise enhanced maximum oxygen consumption $\left(\mathrm{O}_{2}{ }^{\max }\right)$ by roughly $24 \%$ (0.56 [95\% confidence interval, $\left.0.47-0.65\right)$ $\mathrm{L}_{\text {min }}-1$, $(P<0.001)$ with no significant changes in body mass, body composition or fat distribution. Together with energy restriction there was a significant decrease in body mass of $10.1(8.0-12.2) \mathrm{kg}$, lean body mass (LBM) of $2.4(1.5-3.3) \mathrm{kg}$, fat mass (FM) of 7.7 $(5.9-9.6) \mathrm{kg}$, waist-to-hip ratio (WHR) of $0.03(0.01-0.04)$ and the sum of 6 skinfolds of 26.9 (15.4-38.4) mm ( $P<0.001)$. In conclusion, sedentary free-living overweight men, 16 weeks of energy restriction, but not vigorous intensity exercise, resulted in significant drops in body mass, LBM and FM. Also, vigorous intensity exercise when combined with energy restriction changed BF distribution or body composition seen with energy restriction alone.

Another study was designed to compare the effects of intermitted physical activity on weight loss, obedience and fitness, and to examine the effect of mixing intermittent physical activity with that using home exercise equipment [55]. The participants were 148 sedentary, overweight men (mean BMI, 32.8 [4.0] kg/ ${ }^{2}$ ) and women (mean age, 36.7 [5.6] years) in a weight management program. The study consisted of three groups: longbout exercise (LB), multiple short-bout exercise (SB), or multiple SB exercise with home exercise equipment (SBEQ) using a treadmill. In a control behavioral weight program, 115 (78\%) participants completed the program . At 18 months, the mean (SD) weight loss was significantly bigger in participants in the SBEQ group in comparison with the participants in the SB group $-7.4[7.8] \mathrm{kg}$ vs $-3.7[6.6] \mathrm{kg} ; P<0.05)$. The mean (SD) weight loss for the participants in the LB group $(-5.8$ [7.1] $\mathrm{kg}$ ) was not significantly changed compared to the participants in the SB or SBEQ groups. Subjects in the SBEQ group maintained a higher level of exercise than subjects in both the SB and LB groups $(P<$ 
$0.05)$ at 13-18 months of treatment. All groups showed an increase in cardiorespiratory fitness from baseline to 18 months, with no difference between the groups. The mean (SD) weight loss at 18 months was significantly greater in individuals exercising $>200$ $\mathrm{min} / \mathrm{wk}$ throughout the intervention $(-13.1[8.0] \mathrm{kg}$ ) compared with individuals exercising 150-200 min/wk (-8.5 [5.8] kg) or <150 min/wk (-3.5 [6.5] kg) $(P<0.05)$. Compared with the LB group, subjects in the SB group did not experience improved long-term weight loss, exercise participation or cardiorespiratory fitness. Access to home exercise equipment facilitated the maintenance of $\mathrm{SB}$, which improved long-term weight loss. A dose-response connection occurred between amount of exercise and long-term weight loss in overweight adult women.

There have been two studies comparing programs that involve home-based physical activity and those that include supervised exercise programs [27, 56]. In these studies, all patients received the same diet and behavioral instruction and the same exercise goals, but the programs differed in the format used to achieve the physical activity. In both the studies, no difference was noted in the short-term weight loss, however, the maintenance of weight loss was better with home-based exercise in comparison to supervised activity. The advantage of the former was the independence of the choice to exercise, where and the way they individual wanted.

Participation in behavioral weight loss programs is encouraged to gradually increase one's physical activity and avoid injury. The goal for physical activity varies across programs, but often participants are instructed to gradually increase their activity until they achieve a level of at least $1000 \mathrm{kcal} / \mathrm{wk}$. Participants selected the type of exercise they like, but most used walking for the greater part of their activity. It is a common practice that walking 1 mile burns approximately $150 \mathrm{kcal}$ (heavier patients will burn more calories). Otherwise, patients may be given a goal of attaining at least $150 \mathrm{~min} / \mathrm{wk}$ of physical activity, using brisk walking or activities of similar intensity [57].

Lifestyle factors linked to obesity play a key role in the avoidance and therapy of type-2 diabetes. There has been improvement in the development of behavioral approaches to alter these lifestyle behaviors [22]. Further research, however, is clearly needed, because the rates of obesity worldwide are escalating, and changing behavior for the long term has proven to be very difficult. The article by the National Institute of Diabetes and Digestive and Kidney Diseases [58] on behavioral science research in diabetes identifies four key topics related to obesity and physical activity that are of high importance for potential research: (i) environmental factors related to obesity, eating and physical activity; (ii) adoption and maintenance of healthy eating, physical activity and weight; (iii) etiology of eating and physical activity; and (iv) multiple behavior changes. 
Another systematic review [59] results showed that a mean weight loss of 5-8.5 kg was noted throughout the first six months from interventions including a low-calorie diet and/or medication for weight loss with weight maintenance at nearly six months. In studies expanding to 48 months, a mean $3-6 \mathrm{~kg}$ of weight loss was kept with nobody undergoing weight regain to baseline. On the other hand, counselling-alone and exercise-alone participants underwent minimum weight loss. Weight-loss programs with energy restriction and physical activity showed moderate weight loss whereas when medication was added that enhanced the maintenance.

The focus in behavioral programs is typically aerobic exercise, however, Wadden et al. $[56,60]$ studied resistance training and the combination of resistance training and aerobic exercise. They found no difference in weight losses achieved with the various types of exercise.

Data from the National Weight Control Registry highlight the importance of physical activity for weight-loss maintenance [61]. In the NWCR, 91\% of individuals report that physical activity was one aspect of their weight-loss maintenance. Women reported expending an average of $2545 \mathrm{kcal} / \mathrm{wk}$ in exercise and men reported $3293 \mathrm{kcal} / \mathrm{wk}$. These data suggest that successful weight losers exercise over an hour a day. The high exercise level reported by NWCR members has raised the question of whether participants in weight-loss programs should be encouraged to achieve higher activity levels than typically recommended.

In another study, Jakicic et al. [55] reported better weight-loss maintenance in patients who exercised $>200 \mathrm{~min} / \mathrm{wk}$ and Jeffery et al. [62] and Wing et al. [22] found that patients in the highest quartile of exercise, who expended over $2500 \mathrm{kcal} / \mathrm{wk}$, had better weight-loss maintenance than those with lower levels of activity.

A randomized controlled trial [62] comparing $1000 \mathrm{kcal} / \mathrm{wk}$ and $2500 \mathrm{kcal} / \mathrm{wk}$ prescriptions was performed; at month 18 , weight loss in the intensive-exercise group was considerably bigger than the low-exercise group of $6.7 \mathrm{~kg}$ and $4.1 \mathrm{~kg}$, respectively. Therefore, although the original goal of behavioral programs was around $1000 \mathrm{kcal} / \mathrm{wk}$ or $150 \mathrm{~min} / \mathrm{wk}$, it was suggested that patients advanced to higher goals over time.

Jeffery et al. [63] studied the use of personal trainers and financial incentives to increase exercise in a behavioral weight-loss program. In this study, 91 healthy, overweight adult hospital employees aged $18-60$ years with a BMl of $25-36 \mathrm{~kg} / \mathrm{m}^{2}$ were examined. Participants were randomly assigned to a six-month weight loss program of either Internet education (education; $N=32$ with complete data) or Internet behavior therapy (behavior therapy; $N=33$ with complete data). All participants were given one group weight loss session and access to a website with organized links to Internet weight-loss resources. Participants in the behavior-therapy group received additional 
behavioral guidelines, including a sequence of 24 weekly behavioral instructions via e-mail, weekly online submission of self-monitoring diaries with individualized therapist feedback via e-mail and an online bulletin board. Repeated-measures analyses showed that the behavior therapy group lost more weight than the education group $(P=0.005)$. The behavior therapy group lost a mean (SD) of $4.0(2.8) \mathrm{kg}$ by three months and 4.1 (4.5) $\mathrm{kg}$ by six months. More participants in the behavior therapy than education group achieved the $5 \%$ weight loss goal (45\% vs $22 \% ; P=0.05$ ) by 6 months. Changes in waist circumference were also greater in the behavior-therapy group than in the education group at both three $(P=0.001)$ and six $(P=0.005)$ months. Participants in a structured behavioral treatment program with weekly contact and individualized feedback had better weight loss compared with those given links to educational websites.

Participants in behavioral programs were instructed to record all activities they complete. To simplify recording, they are usually coached to base only on exercise sessions that last at least $10 \mathrm{~min}$. Patients recorded either calories used in activity or minutes of activity. Patients in behavioral weight-loss programs were guided to identify these energy-saving devices and plan ways in which they can expend more energy in their daily activities, for example, parking away from the store, using stairs and walking to the nearby shops. Although these lifestyle behavior changes added up over time to significant increases in energy expenditure, they are difficult to quantify and hard to record in self-monitoring diaries. Therefore, such lifestyle activities were examined as a supplement to longer, more structured activity/exercise session. The number one barrier to exercise was lack of time. To address this problem, patients found it easier to exercise for multiple 10-min sessions rather than one 40-min session. In the study of Jakicic et al. [64], 40 patients were assigned to exercise in one 40-min session/day, five days/wk or to complete four 10-min sessions on each of the five days. All other aspects of the weight-loss programs were identical in the two conditions. The short-session program produced better initial adherence and comparable long-term changes in weight and cardiovascular fitness compared to the long-session program. Thus, exercising in multiple sessions may be a useful option for some participants.

Moreover, studies performed on children show that decreasing the number of $\mathrm{hr} / \mathrm{wk}$ of sedentary activities (TV, computer games) may be an effective approach to weight control $[65,66]$. Epstein and colleagues [65] compared the effects of increasing physical activity, decreasing sedentary behavior and the combination of the two in a study of overweight children aged 8-12 years. The children who decrease sedentary time had better long-term weight control outcome and better enhancements in their fitness levels. These results suggest that as the children decreased sedentary activities, they adopted other more physically active pursuits. Maintenance of physical activity is the key for 
long-term weight loss $[49,53]$, however, it is difficult to motivate patients to continue to be physically active in long term. Behavioral programs teach patients strategies for dealing with common barriers to exercise, for example, exercising in hot weather or cold weather; appropriate stretching exercises to prevent injuries. Motivation for physical activity is increased by encouraging patients to do a variety of different activities that they enjoy and helping patients recognize the improvements in fitness that occur with regular exercise.

In a study by Robinson et al. [66], an association was found between television viewing and child and adolescent fattiness. The objective was to assess the effects of reducing television, videotape and video game use on changes in adiposity, physical activity and dietary intake. Two elementary schools participated in a randomized controlled trial with 192 students (mean age, 8.9 years). The children of one of the two schools had an 18-lesson, 6-month classroom curriculum to reduce television, videotape and video game usage. The main outcome measure was BMI. The interventiongroup had statistically significant relative decreases in BMI (intervention vs control change: 18.38-18.67 $\mathrm{kg} / \mathrm{m}^{2} \mathrm{vs} 18.10-18.81 \mathrm{~kg} / \mathrm{m}^{2}$, respectively; adjusted difference $-0.45 \mathrm{~kg} / \mathrm{m}^{2}$ [95\% confidence interval $\{\mathrm{Cl}\},-0.73$ to -0.17 ; $P=0.002$ ), triceps skinfold thickness (intervention vs control change:14.55-15.47 mm vs 13.97-16.46 mm, waistcircumference (intervention vs control change: $60.48-63.57 \mathrm{~cm}$ vs $59.51-64.73 \mathrm{~cm} ; P<0.001$ ) and WHR (intervention vs control change: $0.83-0.83$ vs $0.82-0.84$, respectively; adjusted difference, -0.02 [95\% Cl, -0.03to -0.01]; $P<0.001$ ). Relative to controls, intervention groupchanges were accompanied by statistically significant decreasesin children's reported television viewing and meals eaten infront of the television. There were no statistically significantdifferences between groups for changes in high-fat food intake, moderate-tovigorous physical activity and cardiorespiratoryfitness. In conclusion, reducing television, videotape and videogame use may be a promising, population-based approach to preventchildhood obesity.

The American College of Sports Medicine (ACSM) recommends specific guidelines for exercise-induced weight loss $[67,68]$. The exercise program should promote an expenditure of 300-500 kcal/session and 1000-2000 kcal/wk for adults. However, this may not be possible for the severely obese person. NHLBI [18] guidelines recommend obese patients to start with moderate levels of physical activity for $30-45 \mathrm{~min}, 3-5$ days/wk, working up to exercise sessions on most, and if possible, all days of the week.

The results from studies have demonstrated that exercising alone without an energyrestricted diet program weight loss will be at minimal [49]. Moreover, it is not known whether weight loss was due to exercise alone or whether the participants also altered their dietary intake because they were enrolled in an exercise program. In addition, 
these data may not represent the effect of exercise in obese persons because most subjects enrolled in these studies were slightly overweight men. Vigorous exercise training causes much greater losses in body weight when energy intake is held constant [49].

Based on a study done by Ballor et al. [69], roughly $75 \%$ of weight that is lost by dieting is composed of fat and $25 \%$ is fat-free mass. Adding a physical activity program to dietary therapy can change the composition of weight loss. Two metaanalyses trials [70] published found that exercise can reduce the loss of FFM. In subjects with a mean weight loss of $10 \mathrm{~kg}$, regular exercise decreased the percentage of weight lost as FFM by half, from approximately $28 \%$ to $13 \%$ in men and from $24 \%$ to $11 \%$ in women $(P<0.05)$. However, this large difference in the percentage of weight lost as FFM represented only a small (approximately $1 \mathrm{~kg}$ ) difference in the absolute amount of FFM lost between groups. Moreover, conservation of FFM does not necessarily represent conservation of muscle protein; the greater retention of FFM associated with exercise may be related to increased retention of body water and muscle glycogen. It is not known whether performing resistance exercise while dieting leads to greater conservation of FFM than performing endurance exercise because of limited and conflicting data [56, 71].

Over the last three decades, the American College of Sports Medicine (ACSM) has recommended physical activity and exercise guidelines. While these guidelines have changed dramatically over the years, and while other groups have offered alternatives, the ACSM recommendations remain the core for physical activity programs.

In 1978, the ACSM recommended that individuals exercise three to five days a week for 15 to $60 \mathrm{~min}$, with an overall goal of expending $300 \mathrm{kcal} /$ activity session. The next update of these guidelines occurred in 1990. ACSM retained the aerobic component of its original guidelines but added a strength training recommendation and underscored the importance of realistic, personalized exercise programs. The most recent ACSM recommendations for physical activity were published in 2006 in conjunction with the Centers for Disease Control and Prevention (CDC). At this time, several modifications were made to existing components of the ACSM guidelines. The aerobic component was increased to a minimum of $\mathbf{3 0}$ min of moderate intensity physical activity on most days of the week. Additionally, ACSM recognized that physical activity has an additive effect, and suggested that three 10-min sessions of physical activity could provide health benefits similar to that obtained with one 30 -min session [68].

The 2006 ACSM guidelines remained in use. However, these physical-activity guidelines were not consistently agreed upon due to the introduction of recommendations from other well-regarded scientific groups. For example, the Institute of Medicine (IOM) 
recommended 60 min of moderate intensity physical activity each day. In addition, the President's Council on Physical Fitness and Sports (PCPFS) recommended 20 min of vigorous activity at least three times/wk, whereas the American Heart Association recommended 30-60 min of physical activity 5-7 days/wk. These competing recommendations have created a debate as to which guidelines should be adopted for public health initiatives. Noteworthy health benefits can be attained with moderate amount of physical activity (e.g., 30 min of brisk walking or raking leaves, 15 min of running or 45 min of playing volleyball) on most, if not all, days of the week. It was concluded that by a modest increase in everyday activity, most people can improve their health and quality of life.

There have been several randomized controlled trials comparing the effects on weight loss of diet only, exercise only and the combination [18]. These studies suggest that exercise alone has very small effects on body weight, and that adding exercise to a diet program increases initial weight loss by approximately $2 \mathrm{~kg}$. These modest effects of exercise may well be due to the low dose of exercise used in many of these trials and the short duration of the studies. The greatest benefits of exercise are seen in the maintenance of weight loss. Of the six studies that have examined long-term weight losses in diet only versus diet plus exercise, all six found that the latter had better outcomes, although in many of these studies the difference was not statistically significant [49]. Correlational data are even stronger in suggesting the benefits of longterm physical activity for maintenance of weight loss [72].

\section{Conclusion}

Obesity is a chronic condition with a considerable potential of relapse; the prevalence of obesity internationally has attained epidemic percentages. Consequently, longerterm treatments with the use of NCP are needed. Physical inactivity and poor diet have been acknowledged as primary contributors to the leading causes of death in developed countries. It is unsuccessful that more emphasis is given to calorie-restriction dieting rather than behavioral modification for diet and physical activity in accomplishing and maintaining weight loss, which it is the main reason for relapse. The fact is that integrating appropriate behavioral modification techniques for diet and physical activity into one's life is an essential component of achieving and maintaining a healthy body weight. The NCP is a standardized model intended to guide dietitians in providing high quality nutrition care. The Nutrition Care Model is a graphic visualization that illustrates the steps of the NCP as well as internal and external factors that impact application of 
the NCP. The central component of the model is the relationship of the target client or group and the dietitian.

\section{Acknowledgements}

None.

\section{Funding}

None.

\section{References}

[1] Swinburn B, Dietz W, Kleinert S. A lancet commission on obesity. Lancet. 2015;386 (10005):1716-1717.

[2] World Health Organization. 2018.

[3] Buchan DS, Ollis S, Thomas NE, Baker JS. Physical activity behaviour: An overview of current and emergent theoretical practices. Journal of Obesity. 2012;2012:546459.

[4] Moore GF, Audrey S, Barker M, Bond L, Bonell C, Hardeman W, et al. Process evaluation of complex interventions: Medical Research Council guidance. 2015;350: h1258.

[5] WHO. 1998.

[6] Fontaine K, Redden D, Wang C, Westfall A, Allison D. Years of life due to obesity. JAMA. 2003;289(2):187-193.

[7] Calle E, Rodriguez MD, Walker-Thurmond K, Thun MD. Overweight, obesity, and mortality from cancer in a prospectively studied cohort of US adults. New England Journal of Medicine. 2003;348:1626-1638.

[8] Caballero B. The global epidemic of obesity: An overview. Epidemiologic Reviews. 2007;29(1):1-5.

[9] National Heart, Lung and Blood Institute, National Institute of Diabetes and Digestive and Kidney Disease. Clinical guidelines on the identification, evaluation and treatment of overweight and obesity in adults - The evidence report. Obesity Research.1998;6(2):51S-209S.

[10] Purnell JQ. Endotext [Internet]. Feingold KR, Anawalt B, Boyce A, Chrousos G, de Herder WW, Dhatariya K, Dungan K, et al., editors. South Dartmouth (MA): MDText.com, Inc.; 2018. Definitions, classification, and epidemiology of obesity NCB. 
[11] World Health Organization. 2017.

[12] International Obesity Task Force, 2009.

[13] Andreou E, Hadjigeorgiou P, Kyriakou K, Avraam T, Chappa G, Kallis P, et al. Risk factors of obesity in a cohort of 1001 Cypriot adults: An epidemiological study. Hippokratia. 2012;16(3):256-260.

[14] Martin G, Pear J. Behaviour modification: What it is and how to do it. 8th ed. Upper Saddle River, NJ: Pearson Prentice Hall; 2007.

[15] Foster-Schubert KE, Alfano CM, Duggan CR, Xiao L, Campbell KL, Kong A, et al. Effect of diet and exercise, alone or combined, on weight and body composition in overweight-to-obese post-menopausal women. Obesity. 2012;20(8):1628-1638.

[16] Vogel T, Brechat P, Leprêtre P, Kaltenbach G, Berthel M, Lonsdorfer J. Health benefits of physical activity in older patients: a review. International Journal of Clinical Practice. 2009;63(2):303-320.

[17] Welk BE, Blair S. Fitnessgram reference guide: Health benefits of physical activity and fitness in children. Dallas TX: The Cooper Institute; 2001.

[18] National Heart, Lung, and Blood Institute. Detection, evaluation, and treatment of high blood cholesterol in adults (Adult Treatment Panel III). Revista Panamericana de Salud Publica. 2001;9(5):338-344.

[19] Lacey and Pritchett. 2003.

[20] Lee SY, Kim J, Oh S, Kim YM, Woo S, Jang HB. A 24-week intervention based on nutrition care process improves diet quality, body mass index, and motivation in children and adolescents with obesity. Nutrition Research. 2020;84:53-62.

[21] Chen J, Gemming L, Hanning R, Allman-Farinelli M. Smartphone apps and the nutrition care process: Current perspectives and future considerations. Patient Education and Counseling. 2018;101(4):750-757.

[22] Wing RR. Handbook of obesity. Bray GA, Bouchard C, James WP, editors. New York: Marcel Dekker; 1998. Behavioural approaches to the treatment of obesity; p 855873.

[23] Lichtman SW, Pisarska K, Berman ER, Pestone M, Dowling H, Offenbacher E, et al. Discrepancy between self-reported and actual caloric intake and exercise in obese subjects. The New England Journal of Medicine. 1992;327(27):1893-1898.

[24] Middleton K, Anton S, Perri M. Long-term adherence to health behavior change. American Journal of Lifestyle Medicine. 2013;7(6):395-404.

[25] Fabricatore AN, Wadden TA, Moore RH, Butryn ML, Gravallese EA, Erondu NE, et al. Attrition from randomized controlled trials of pharmacological weight loss agents: a systematic review and analysis. Obesity Reviews. 2009;10(3):333-341. 
[26] Foreyt J, Goodrick K. Evidence for success of behaviour modification in weight loss and control. Annals of Internal Medicine. 1993;119(7 Pt 2):698-701.

[27] Perri MG, Martin AD, Leermakers EA, Sears SF, Notelovitz M. Effects of group- versus home-based exercise in the treatment of obesity. Journal of Consulting and Clinical Psychology. 1997;65(2):278-285.

[28] Dubbert PM, Wilson GT. Goal-setting and spouse involvement in the treatment of obesity. Behaviour Research and Therapy. 1984;22:227-242.

[29] Kayman S, Bruvold W, Stern JS. Maintenance and relapse after weight loss in women behavioral aspects. The American Journal of Clinical Nutrition. 1990;52(5):800-807.

[30] Hartman W, Wapner D, Saxton J. A simple procedure to identify persons at risk for dieting failure. Paper presented at: 24th Annual Conference, Association for Advancement of Behavior Therapy; 1990. San Francisco, United States.

[31] Rodin J. The current status of the internal-external obesity hypothesis. American Psychologist. 1981;36(4):361-372.

[32] Rodin J, Elias M, Silberstein L, Wagner A. Combined behavioral and pharmacologic treatment for obesity: Predictors of successful weight maintenance. Journal of Consulting and Clinical Psychology. 2002;56(3):399-404.

[33] Wilfley D, Schreiber G, Pike K, Striegel-Moore R, Wright D, Rodin J. Eating disturbance and body image: A comparison of a community sample of adult black and white women. International Journal of Eating Disorders. 1998;20(4):377-387.

[34] Jeffery WR. Financial incentives and weight control. Preventive Medicine. 2011;55(Suppl):S61-S67.

[35] Spiegel TA, Wadden TA, Foster GD. Objective measurement of eating rate during behavioral treatment of obesity. Behaviour Therapy. 1991;22(1):61-67.

[36] Spitzer RL, Devlin M, Walsh BT, Hasin D, Wing R, Marcus M. Binge eating disorder: A multisite field trial of the diagnostic criteria. International Journal of Eating Disorders. 1993;11(3):191-203.

[37] Telch CF, Agras WS, Rossiter EM, Wilfley D, Kenardy J. Group cognitive-behavioral treatment for the nonpurging bulimic: An initial evaluation. Journal of Consulting and Clinical Psychology. 1990;58(5):629-635.

[38] Kelley CP, Sbrocco G, Sbrocco T. Behavioral modification for the management of obesity. Primary Care. 2016;43(1):159-175.

[39] Philippou C, Andreou E, Menelaou N, Hajigeorgiou P, Papandreou D. Effects of diet and exercise in 337 overweight/obese adults. Hippokratia Journal. 2011;16(1):449_ 453. 
[40] National Task Force on the Prevention and Treatment of Obesity (NTFPTO). National task force on the prevention and treatment of obesity. NTFPTO; 2003.

[41] Wing RR, Marcus MD, Blair EH, Burton LR. Psychological responses of obese type II diabetic subjects to very-low-calorie diet. Diabetes Care. 1991;14(7):596-599.

[42] Schlundt DG, Hill JO, Pope-Cordle J, Arnold D, Virts KL, Katahn M. Randomized evaluation of a low fat ad libitum carbohydrate diet for weight reduction. International Journal of Obesity. 1993;17(11):623-629.

[43] Pascale RW, Wing RR, Butler BA, Mullen M, Bononi P. Effects of a behavioral weight loss program stressing calorie restriction versus calorie plus fat restriction in obese individuals with NIDDM or a family history of diabetes. Diabetes Care. 1995;18(9):1241-1248.

[44] Hays N, Sullivan D, Fluckey JD. Effects of an ad libitum, high carbohydrate diet and aerobic exercise training on insulin action and muscle metabolism in older men and women. The Journals of Gerontology, Series A: Biological Sciences and Medical Sciences. 2006;61(3):299-304.

[45] Holden JH, Darga LL, Olson SM, Stettner DC, Ardito EA, Lucas CP. Long-term followup of patients attending a combination very-low calorie diet and behaviour therapy weight loss programme. International Journal of Obesity. 1992;16(8):605-613.

[46] Harris JK, French SA, Jeffery RW, McGovern PG, Wing RR. Dietary and physical activity correlates of long term weight loss. Obesity Research. 1994;2(4):307-313.

[47] Wing RR, Hill JO. Successful weight loss maintenance. Annual Review of Nutrition. 2001;21:323-341.

[48] Thompson WG, Cook DA, Clark MM, Bardia A, Levine JA. Treatment of obesity. Mayo Clinic Proceedings. 2007;82(1):93-102.

[49] Wing R. Physical activity in the treatment of the adulthood overweight and obesity, current evidence and research issues. Medicine \& Science in Sports \& Exercise. 1999;31(11):S547-S552.

[50] Metz JA, Stern SS, Kris-Etherton P. A randomized trial of improved weight loss with a prepared meal plan in overweight and obese patients. Archives of Internal Medicine. 2000;160(14):2150-2158.

[51] Jeffery RW, Wing RR, Thorson C, Burton LR, Raether C, Harvey J, et al. Strengthening behavioural interventions for weight loss. A randomized trial of food provision and monetary incentives. Journal of Consulting and Clinical Psychology. 1993;61(6):10381045.

[52] Varkevisser RDM, van Stralen MM, Kroeze W, Ket JCF, Steenhuis IHM. Determinants of weight loss maintenance: A systematic review. Obesity Review. 2018;20(2):171-211. 
[53] Pronk NP, Wing RR. Physical activity and long-term maintenance of weight loss. Obesity Research. 1994;2(6):587-599.

[54] Tate DF, Wing RR, Winett RA. Using internet technology to deliver a behavioral weight loss program. JAMA. 2001;285(9):1172-1177.

[55] Jakicic J, Wing R, Winters C. Effects of intermittent exercise and use of home exercise equipment on adherence, weight loss, and fitness in overweight women. JAMA. 1999;282(16):1554-1560.

[56] Wadden TA, Vogt RA, Andersen RE, Bartlett SJ, Foster GD, Kuehnel RH. Exercise in the treatment of obesity: Effects of four interventions on body composition, resting energy expenditure, appetite, and mood. Journal of Consulting and Clinical Psychology. 1997;65(2):269-277.

[57] Grave D, Calugi S, Centis E, Ghoch M, Marchesini G. Cognitive-behavioral strategies to increase the adherence to exercise in the management of obesity. Journal of Obesity. 2011;2011:348293.

[58] National Institute of Diabetes and Digestive and Kidney Diseases. 2001.

[59] Franz M, VanWormer J, Crain L, Boucher J, Histon T, Caplan W, et al. Weightloss outcomes: A systematic review and meta-analysis of weight-loss clinical trials with a minimum 1-year follow-up. Journal of the American Dietetic Association. 2007;107(10):1755-1767.

[60] Wadden TA, Vogt RA, Foster GD, Anderson DA. Exercise and maintenance of weight loss, 1-year follow-up of a controlled clinic trial. Journal of Consulting and Clinical Psychology. 1998;66(2):429-433.

[61] Klem ML, Wing RR, McGuire MT, Seagle HM, Hill JO. A descriptive study of individuals successful at long term maintenance of substantial weight loss. The American Journal of Clinical Nutrition. 1997;66(2):239-246.

[62] Jeffery RW, Wing RW. The effects of an enhanced exercise program on long-term weight loss. Obesity Research. 2001;9:100S

[63] Jeffery RW, Wing RR, Thorson C, Burton LC. Use of personal trainers and financial incentives to increase exercise in a behavioural weight-loss program. Journal of Consulting and Clinical Psychology. 1998;66(5):777-783.

[64] Jakicic JM, Wing RR, Butler BA, Robertson RJ. Prescribing exercise in multiple short bouts versus one continuous bout: Effects on adherence, cardiorespiratory fitness, and weight loss in overweight women. International Journal of Obesity. 1995;19(12):893-901. 
[65] Epstein LH, Valoski AM, Vara LS, McCurley J, Wisniewski L, Kalarchian MA, et al. Effects of decreasing sedentary behavior and increasing activity on weight change in obese children. Health Psychology. 1995;14(2):109-115.

[66] Robinson T. Reducing children's television viewing to prevent obesity. JAMA. 1999;282(16):1561-1567.

[67] Mahler DA. Guidelines for exercise testing and prescription. 6th ed. Baltimore, MD: American College of Sports Medicine; 1999.

[68] Whaley $\mathrm{MH}$, Otto RM, Brubaker PH. ACSM's guidelines for exercise testing and prescription. 7th ed.Philadelphia, PA, United States: Lippincott Williams \& Wilkins; 2006.

[69] Ballor DL, Poehlman ET. Exercise-training enhances fat-free mass preservation during diet-induced weight loss: a meta-analytical finding. International Journal of Obesity and Related Metabolic Disorders. 1994;18(1):35-40.

[70] Garrow et al. 1995.

[71] Geliebter A, Maher MM, Gerace L. Effects of strength or aerobic training on body composition, resting metabolic rate, and peak oxygen consumption in obese dieting subjects. American Journal of Clinical Nutrition. 1997;66(3):557-563.

[72] Catenacci V, Wyatta $\mathrm{H}$. The role of physical activity in producing and maintaining weight loss. Nature Clinical Practice Endocrinology \& Metabolism. 2007;3(7):518529. 